\title{
Distorted mass edges at LHC from supersymmetric leptoquarks
}

\author{
Jürgen Reuter* and Daniel Wiesler ${ }^{\dagger}$ \\ DESY Theory Group, Notkestrasse 85, D-22603 Hamburg, Germany; University of Edinburgh, \\ School of Physics and Astronomy, JCMB, The King's Buildings, Mayfield Road, Edinburgh EH9 3JZ, Scotland; \\ and Albert-Ludwigs-Universität Freiburg, Physikalisches Institut, Hermann-Herder-Strasse 3, D-79104 Freiburg, Germany
} (Received 27 October 2010; revised manuscript received 18 May 2011; published 21 July 2011)

\begin{abstract}
Supersymmetric (SUSY) grand unified theories based on exceptional gauge groups such as $E_{6}$ have recently triggered a lot of interest. Aside from top-down motivations, they contain phenomenologically interesting states with leptoquark quantum numbers. Their SUSY partners, leptoquarkinos, will appear similar to all $R$-odd particles in decay cascades, but mass edges in kinematic distributionsoriginating from the same semiexclusive final states-will however have major differences to the corresponding edges of ordinary squarks. This distortion of standard observables bears the opportunity to detect them at the LHC, but may also pose significant confusion of underlying model assumptions, which should be handled with care and, if interpreted falsely, might even prevent a possible discovery.
\end{abstract}

DOI: 10.1103/PhysRevD.84.015012

PACS numbers: 14.80.Sv, 12.10.Dm, 12.60.Jv, 13.85.Rm

\section{INTRODUCTION}

Supersymmetry is one of the most promising solutions of the hierarchy and fine-tuning problem, namely, the vast difference between the electroweak (EW) and the Planck scale, and the very stability of this difference. It yields a mechanism for radiatively generating EW symmetry breaking, allows for an exact unification of all forces, and conveys a candidate for dark matter. However, it comes with the price of having new problems, connected to the flavor sector, the stability of the proton, and new sorts of hierarchy problems known as the $\mu$ problem and doublettriplet splitting. To address these questions, models have been developed that derive from a Planck or grand unified theory (GUT) scale exceptional gauge group such as $E_{6}$ [1], and might be embedded in the context of the heterotic string. Such $E_{6}$-based models have a matter-Higgs unification, are automatically anomaly free, include the righthanded neutrino, and solve the $\mu$ problem as an effective next-to-minimal supersymmetric (SUSY) standard model (NMSSM). However, one either has to solve a problem similar to doublet-triplet splitting, or use e.g. an intermediate left-right symmetric model, where the high-scale braking happens through orbifold compactifications $[2,3]$. The fundamental representation of $E_{6}$, the 27, contains exotic states which carry both lepton and baryon numbers and hence act as lepto- or diquarks. This is why a mechanism as in [2] is chosen specifically to prevent a phenomenologically disastrous rapid proton decay. As these exotics are left-chiral superfields (with vectorlike quantum numbers with respect to the EW gauge group), they come as a pair of scalars, $D$ and $D^{*}$, being $R$ even, and a Dirac fermion, $\tilde{D}$, being $R$ odd, at the EW scale. The states are called

\footnotetext{
*juergen.reuter@desy.de

†daniel.wiesler@desy.de
}

leptoquarks and leptoquarkinos, respectively. Their potential discovery at the Large Hadron Collider (LHC) may allow for a direct handle on the GUT structure of these models at the $\mathrm{TeV}$ scale beyond superprecise extrapolation of parameters over 13-15 orders of magnitude.

For the rest of this paper, it is sufficient to take the model-building setup above as a rough motivation of how such states could come about in nature, and further on just assume their existence together with the spectrum of an NMSSM-like model. The phenomenology of the scalar leptoquarks is very similar to that of nonsupersymmetric states due to their $R$-even nature and will be discussed in a following publication [3]. While the pair production of the fermionic superpartners, the leptoquarkinos, is almost completely determined by QCD, their decays as $R$-odd particles show the very same cascadelike structures as squark and gluino decays. However, their decay products contain both nonvanishing lepton and baryon numbers. Hence, kinematic edge structures for the mass determination of new physics states derived from jet-lepton or jet-dilepton exclusive final states have very characteristic features which-using invalid assumptions about the underlying SUSY model—could lead to wrong particle identifications and mass determinations (the latter point is particularly relevant, if the scalar states which happen to be usually heavier than the fermions might lie outside the kinematic reach of the LHC). The intent of this paper is to first introduce the relevant observables before a brief discussion of the exotic fermion production and decay mechanisms is followed by a dedicated study on the impact of leptoquarkinos with varying masses onto SUSY squark analysis methods. Here it is the goal to show the essential and important differences between standard SUSY squark cascades and leptoquarkino-triggered cascades, and why it is important to consider at least both, if not all, possible model alternatives. 


\section{LHC MASS EDGES FROM LEPTOQUARKINOS}

Mass edge variables [4-8] have mostly been developed with a certain decay pattern in mind: left-handed squark into a quark, two leptons and the lightest neutralino via the on-shell decays of the second-to-lightest neutralino and a right-handed slepton:

$$
\tilde{q}_{L} \rightarrow q \tilde{\chi}_{2}^{0} \rightarrow q l^{ \pm} \tilde{l}_{R}^{+} \rightarrow q l^{ \pm} l^{\mp} \tilde{\chi}_{1}^{0} .
$$

Since one is not able to distinguish experimentally which of the leptons $l^{ \pm}$and $l^{\mp}$ is nearest to the quark (in terms of the decay cascade), two specific observables have been introduced [5] to allow for discrimination:

$$
\begin{aligned}
& m_{q l, \text { high }}=\max \left\{m_{q l^{+}}, m_{q l^{-}}\right\}, \\
& m_{q l \text { low }}=\min \left\{m_{q l^{+}}, m_{q l^{-}}\right\} .
\end{aligned}
$$

As squarks are pair produced at the LHC, they decay via the above or even simpler patterns leading to final states that may be able to mimic those of the leptoquarkino signals: two hard partonic jets, two or more opposite sign, same flavor (OSSF) leptons and most importantly large amounts of missing transverse energy. However, it is crucial to note that due to the general assumption of minimal flavor violation in the SUSY lepton sector, flavor is a conserved quantum number and runs like a line through the cascade chain. Hence, OSSF leptons originating from squark decays inside a single decay chain are (up to a simple combinatorical factor) equally likely than opposite sign different flavor (OSDF) lepton contributions for standard MSSM-type signals, since the branching ratios into first and second generation fermion/sfermion combinations are of the same size in most mSUGRA scenarios. These OSDF signals are coming in the standard MSSM paradigm from different flavor decays of pair-produced SUSY particles. They are, however, not present in the case of

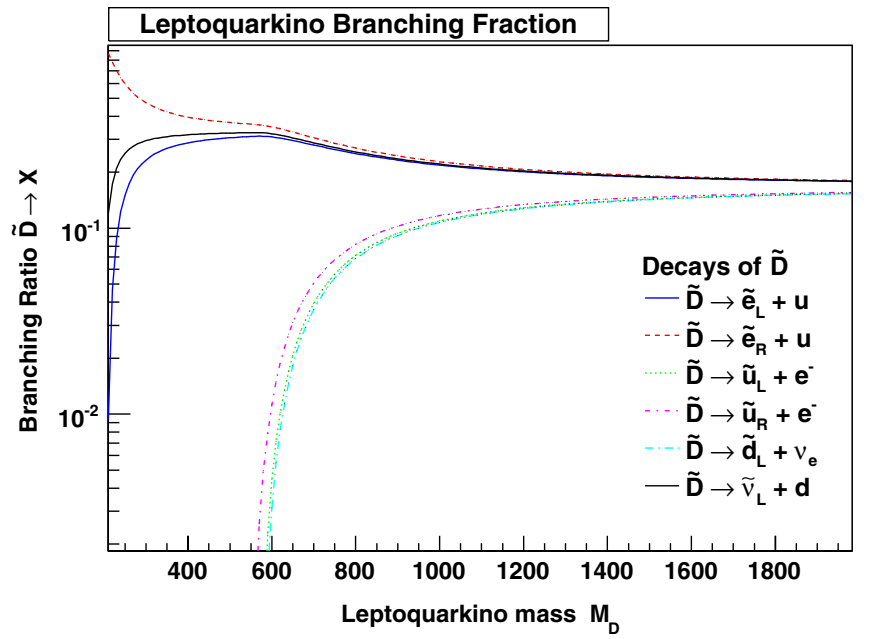

leptoquarkino decays, when we consider flavor-diagonal Yukawa couplings and standard flavor-changing neutralcurrent constraints, which allows one to suppress large amounts of standard SUSY "backgrounds."

As a selection criterion, we accept events only if they have exactly two hard jets with a minimum $p_{T}$ of $50 \mathrm{GeV}$. This requirement is intended to suppress one additional collinear initial state radiation jet, for allowed jet multiplicities of three. If we also choose to take jet multiplicities of four into account, we have to be more careful: to avert the contamination by e.g. a gluino pair production signal, we need the third hardest jet to have a maximum $p_{T}$ of $50 \mathrm{GeV}$, while retaining the cuts above for the two hardest jets. In the cases investigated here this was sufficient to separate leptoquarkino signals from gluino pair production; however, there is still the possibility from mixed gluino-squark production which is known to be able to be accompanied by possibly harder QCD radiation jets [9]. Furthermore, it could also appear that jets from decays could be rather soft (e.g. in SPS1a where gluinos and squarks are quite degenerate), leading to a confusion with QCD radiation jets. For leptoquarkino signals this seems not to be overly likely (because the leptoquarkino renormalization group equations are not so tightly linked as those of squarks and gluinos), but is nevertheless possible. For the further analysis presented here, we just take the rather simplified cut above and assume that the jet backgrounds are under control. A complete background study is beyond the intent of the present study and is not performed here.

Leptoquarkinos, if existent, are abundantly produced at the LHC, since they are massive colored isosinglet fermions [1]. The leading order cross sections basically depend only on the mass for pair production, and the Yukawa coupling for single production, respectively (see the right-hand side (rhs) of Fig. 1). The Yukawa coupling is

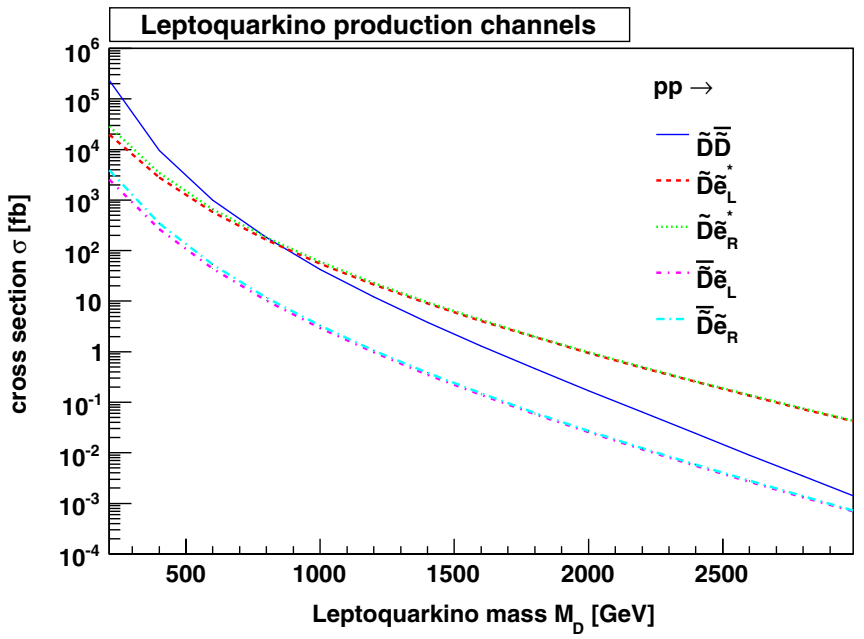

FIG. 1 (color online). Branching ratio for the leptoquarkino decay into fermion and scalar (left panel); leading order cross sections for single and pair production at $14 \mathrm{TeV}$ (right panel). 
without knowledge of the complete GUT model arbitrary, but was taken here to be of the size of the electromagnetic coupling $(y=0.312)$. The left-hand side (lhs) of Fig. 1 shows the branching fractions of the decaying leptoquarkino (for varying masses) into a fermion/sfermion pair. As the decay into squarks and leptons is kinematically forbidden for low leptoquarkino masses (and still heavily phase-space suppressed for increasing leptoquarkino masses), the sleptons dominate in that case as intermediate states in cascades. Though this depends on the parameter space of the models, we follow this assumption from here on. Consequently, a typical leptoquarkino decay may be given by

$$
\tilde{D} \rightarrow q \tilde{l}_{R / L}^{-} \rightarrow q l^{-} \tilde{\chi}_{1}^{0}
$$

whereas a second-to-lightest neutralino in the decay chain starts to become important for heavier masses, leading to different intermediate states, e.g.,

$$
\tilde{D} \rightarrow \tilde{q}_{R / L}^{-} l^{-} \rightarrow q l^{-} \tilde{\chi}_{2}^{0} \rightarrow q l^{-} l^{ \pm} l^{\mp} \tilde{\chi}_{1}^{0} .
$$

Leptoquarkinos produced in pairs thus show the same exclusive final states as squarks (cf. Fig. 2), namely, two hard partonic jets, two or more leptons, and large missing transverse energy in the detector, accounting for the undetected neutralinos. The influence of the second type of cascade (5) however is of less importance, since the new Majorana decay gives rise to additional OSDF lepton contributions, which is absent in the first type of leptoquarkino chain. Additionally, heavier intermediate states are strongly phase-space suppressed and only start to become more important for increasing exotic masses. Consequently, we focus our analysis on the first cascade (4) with the case of only two OSSF leptons present, as it proves to be the most common exotic decay pattern for the regions in parameter space with relatively light leptoquarks.
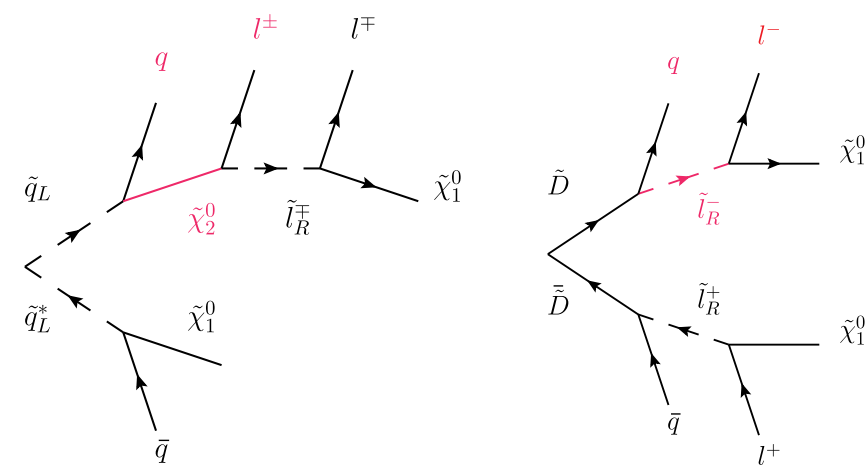

FIG. 2 (color online). Examples for decay cascades under investigation: squark (left panel) and leptoquarkino (right panel) pair production.
At this early partonic stage, standard analysis methods for lepton-quark mass edges applied to events with dedicated leptoquarkino cascades show strong discrepancies to well-known results from standard SUSY signals. The difference emerges due to the intermediate on-shell scalar (squark or slepton) between the quark and lepton compared to a Majorana fermion as e.g. the neutralino in the MSSM: there are no possible spin correlations between lepton and quark, as they are connected through a scalar propagator. As a result, their invariant mass spectrum is equivalent to the dilepton spectrum in standard MSSM models (stemming from a scalar slepton propagator), in that it linearly rises from zero to its maximum at the end point, where it instantly falls down to zero. This case is not necessarily a unique feature of SUSY as there are other models with a scalar propagator, which may distort the actual shape of the relevant invariant mass variables [e.g., in universal extra dimensions (UED), for a general review, cf. [10]]. However, we take the leptoquarkino setup as an application prototype and investigate how such deviations from possible intermediate scalars can arise in the context of a SUSY decay chain. Complete knowledge of the particular extended SUSY model containing leptoquarkinos is not needed for the present study, but will be discussed in a future publication, which in addition will discuss a set of discrete self-consistent parameter points [3]. The generic properties of leptoquarkinos, however, could be inferred rather independent of the underlying model setup: they are scalar particles which decay into final states with lepton and baryon number, but because of the assumption of conserved $R$ parity do not show a peak in the corresponding invariant mass distribution. The smoking-gun signatures are the specific quark-lepton mass edges to be described below in detail, which deviate from standard edges in SUSY squark and gluino searches. (Such decays could indeed arise from squarks in SUSY models with $R$ parity violation; however, due to constraints from proton lifetime these would be overwhelmingly washed out by the standard SUSY decays.) The flavor structure of leptoquarkinos might be quite interesting, especially when considering third-generation leptoquarkinos involving (s)tops, (s) bottoms (experimentally problematic because of the neutrinos in the final state), and (s)taus, but is rather model dependent and thus we postpone a more comprehensive analysis to [3].

For the comparison of ordinary squark with leptoquarkino cascades we are exemplarily using the parameter point SPS1a [11] for the MSSM as well as a model containing leptoquarks ${ }^{1}$ and -inos with varying masses augmented by squarks and sleptons with the same masses as the SPS1a data point $\left(m_{\tilde{u}_{L}}=567 \mathrm{GeV}, m_{\tilde{u}_{R}}=547 \mathrm{GeV}\right.$,

\footnotetext{
${ }^{1}$ The scalars are considered heavier than fermions (masses well above $1 \mathrm{TeV}$ ), since this is usually the case and their presence would most likely alter the shape in that a resonant peak structure would dominate the spectrum.
} 

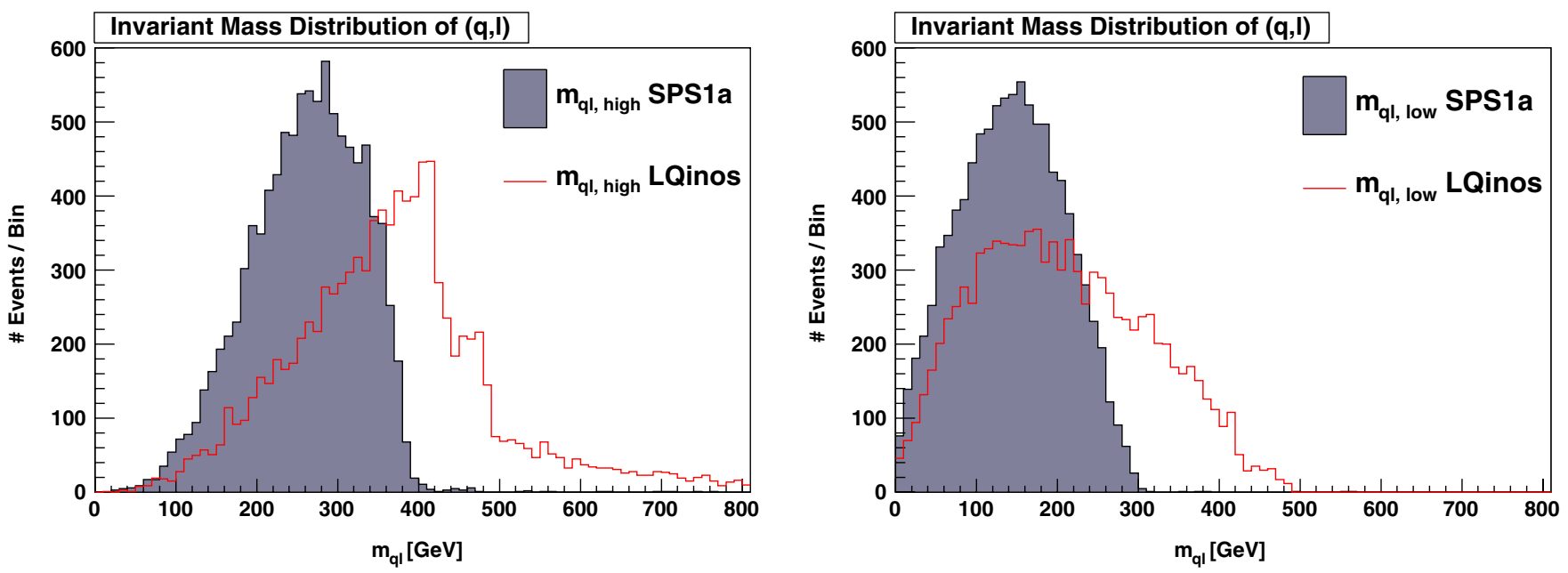

FIG. 3 (color online). Anatomy of leptoquarkino mass edges for $m_{q l \text {,high }}$ and $m_{q l \text { low }}$ with $m_{D}=600 \mathrm{GeV}$.
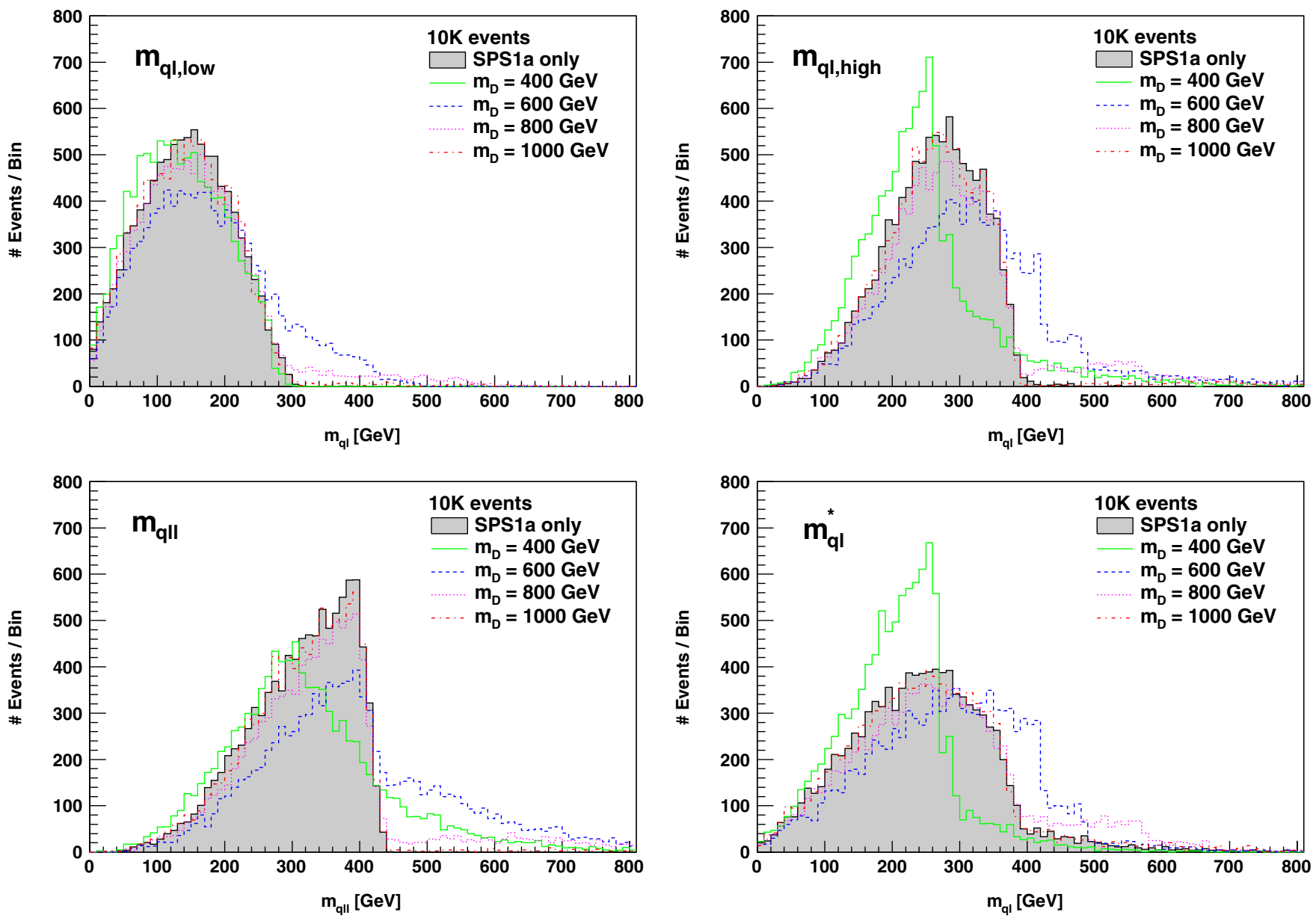

FIG. 4 (color online). Leptoquarkino mass scans between 400 and $1000 \mathrm{GeV}$ show deviations from standard SUSY observables in an underlying SPS1a spectrum. The upper left and right figures disclose the quark-lepton invariant mass spectra of $m_{q l, \text { low }}$ and $m_{q l \text {,high }}$, respectively. The lower parts show the complete quark-dilepton spectrum $m_{q l l}$ on the left and the combinatorics-free $m_{q l}^{*}$ on the right, which is defined in Eq. (7). Continuous, dashed, dotted, and dash-dotted lines correspond to leptoquarkino masses of 400, 600, 800, and $1000 \mathrm{GeV}$, respectively. 

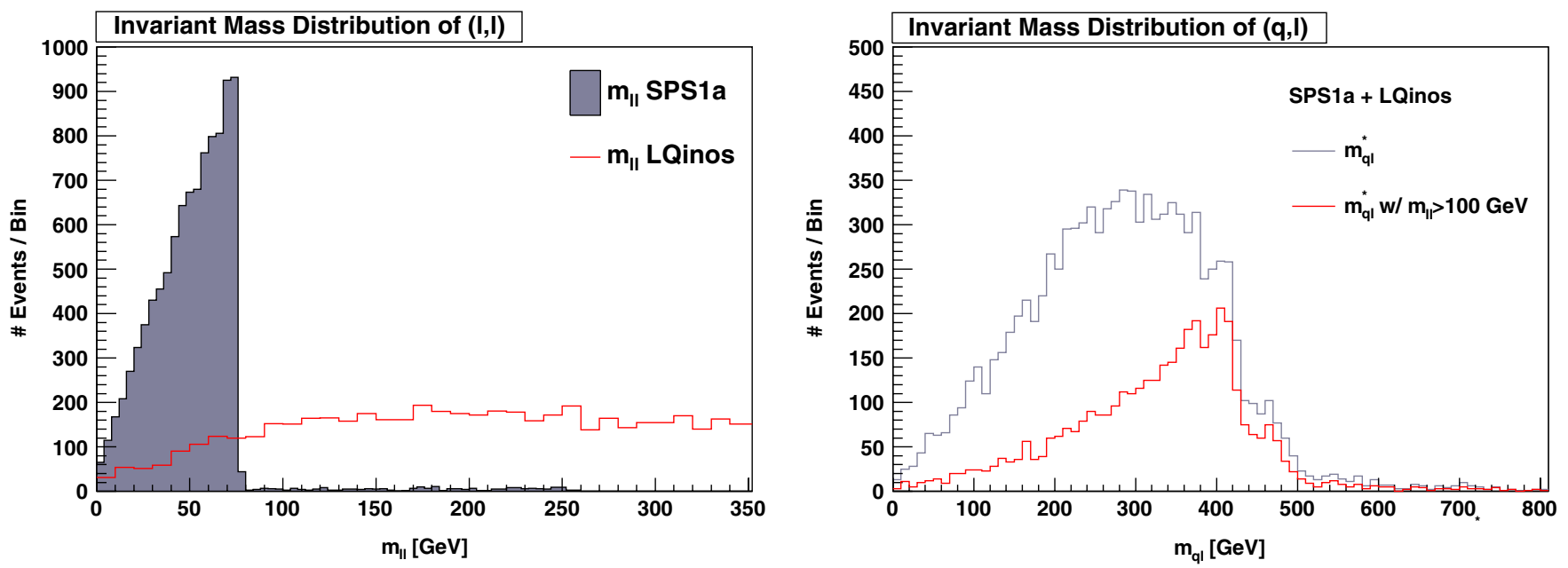

FIG. 5 (color online). The lhs shows the dileptonic spectrum with strong discrepancies between pure SPS1a squark and leptoquarkino signals in that the correlation of both leptons from one specific decay chain is obvious in the first case. This can be used to severely suppress the standard SUSY signals by cutting on $m_{l l}$ shortly above the kinematical edge. The rhs presents the impact of this cut on the variable defined in Eq. (7) $\left(m_{D}=600 \mathrm{GeV}\right)$.

$m_{\tilde{l}_{L}}=204 \mathrm{GeV}, m_{\tilde{l}_{R}}=145 \mathrm{GeV}, m_{\tilde{\chi}_{1}^{0}}=97 \mathrm{GeV}$, and $\left.m_{\tilde{\chi}_{2}^{0}}=181 \mathrm{GeV}\right)$. To reduce the dependency of the effect of edge distortion on the SUSY scenario, we also looked at three other heavy Snowmass Points and Slopes (SPS) spectra including one gauge-mediated supersymmetry breaking (GMSB) case (SPS1b, SPS3, and SPS7 [11]), the results of which are summarized in the Appendix. In general, we find that the following analysis does not depend on the SUSY breaking scenario or the detailed specifics of the chosen parameter point.

The kinematical end points under consideration are given by the masses of the intermediate and mother particles:

$$
\begin{aligned}
m_{q l}^{\max } & =\left[\frac{\left(m_{\tilde{e}_{R(L)}}^{2}-m_{\tilde{\chi}_{1}^{0}}^{2}\right)\left(m_{D}^{2}-m_{\tilde{e}_{R(L)}}^{2}\right)}{m_{\tilde{e}_{R(L)}}^{2}}\right]^{1 / 2} \\
& =433(496) \mathrm{GeV}
\end{aligned}
$$

for a leptoquarkino mass of $600 \mathrm{GeV}$, where the value in parentheses is given for an intermediate left-handed slepton, which is slightly phase-space suppressed in this particular SPS1a scenario. The overall signal consists of the sum of both contributions leading to the shape visible in Figs. 3-5. For each model, a data set of $10 \mathrm{~K}$ unweighted events was generated using a hard-coded implementation of these $E_{6}$-inspired SUSY models into the event generator WHIZARD [12], which is particularly well suited for the LHC beyond the SM studies [13]. While a complete validation of the model implementation using the WHIZARD interface to the FEYNRULES package [14] is under way, the part of the implementation relevant for this paper has been extensively tested.
Returning to the cascade, there still remains the problem of observability: experimentally there is no possibility to select the correct partonic jet and corresponding lepton, which are then to be combined to the invariant mass spectrum. While in MSSM models this would come about due to the presence of the Majorana fermion decay into two OSSF leptons, in the leptoquarkino case two OSSF leptons are to be collected from different cascades, one originating from the leptoquarkino and the other from its antiparticle, respectively. The observables $m_{q l \text { low }}$ and $m_{q l \text {,high }}$, shown in Fig. 3, thus display the tremendous discrepancy, especially the latter one with its sharply falling edge shape, intrinsic to the nature of the scalar intermediate sparticle. The long tail, inherent in the signal variant of $m_{q l, \text { high }}$, is another unusual feature and a direct consequence of the combination of final state particles from two different cascade sides.

This issue of combinatorics however may be addressed by combining the softest jet and the hardest lepton to form an invariant mass spectrum in a single event. This has proven to be useful [15] in terms of resembling the actual shape and thus the most accurate position of the theoretical edge:

TABLE I. Significance estimates for $100 \mathrm{fb}^{-1}$ and the relevant (non-)standard SUSY final state of two hard jets, two OSSF leptons, and $E_{T}$. We consider only standard SUSY events as possible backgrounds.

\begin{tabular}{rcrc}
\hline \hline$m_{D}$ & $N(L Q$ ino $) \& N($ SUSY $)$ & $N_{\text {cut }}$ & $S / \sqrt{S+B}$ \\
\hline 400 & 8763 & 5061 & 54 \\
600 & 1355 & 540 & 15 \\
800 & 684 & 102 & 4 \\
1000 & 594 & 24 & 1 \\
\hline \hline
\end{tabular}



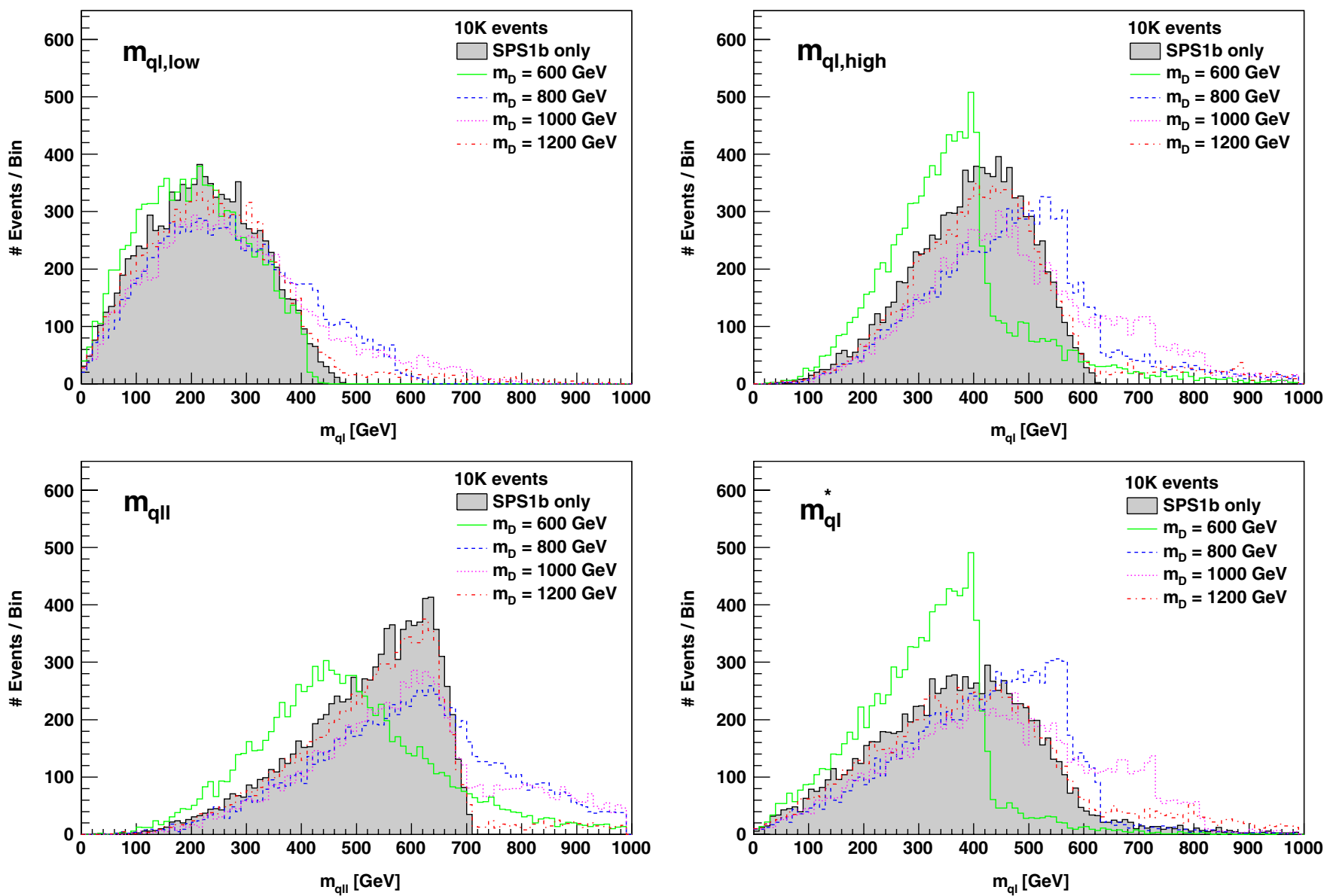

FIG. 6 (color online). The same variables as in Fig. 4, instead dressed with an underlying SPS1b. Continuous, dashed, dotted, and dash-dotted lines correspond to leptoquarkino masses of 600, 800, 1000, and $1200 \mathrm{GeV}$, respectively.

$$
m_{q l}^{*}=m\left(\min _{E}\left\{j_{1}, j_{2}\right\}, \max _{E}\left\{l^{+}, l^{-}\right\}\right)
$$

This observable is analyzed in Fig. 4 for SPS1a (the other SPS scenarios are found in Figs. 6-8) together with $m_{q l \text {,high }}$, $m_{q l \text { low }}$, and $m_{q l l}$ for four different leptoquarkino masses ranging from 400 to $1000 \mathrm{GeV}$ embedded into an underlying SPS1a spectrum. Since the hadronic leading order cross section for pair-produced leptoquarkinos is of the order of $10^{4} \mathrm{fb}$ for masses as low as $400 \mathrm{GeV}$ (see Fig. 1), it dominates the shape of the observables in Fig. 4. The specific shapes of the observables directly hint toward new, possibly exotic physics beyond a standard SUSY paradigm. However, this behavior changes for heavier leptoquarkinos: the higher the mass and thus the lower the cross section of the involved leptoquarkino, the weaker the effect on the observables. While for masses of $600 \mathrm{GeV}$ the shape of the distribution is still predominantly given by the leptoquarkino contribution, differences to squark signals are merely visible for $800 \mathrm{GeV}$ and are practically invisible for $1000 \mathrm{GeV}$ (or will be so after detector effects). These characteristics do not change drastically for the other SPS spectra but are instead shifted to higher values of the leptoquarkino mass. ${ }^{2}$ The feature of edge distortion persists in all of the different SUSY scenarios and is thus mainly independent of the latter, as long as the leptoquarkino mass is not much heavier than the rest of the spectrum.

Given that $m_{q l, i}(i=$ high, low $)$ and $m_{q l l}$ are single $\operatorname{sided}^{3}$ variables, the inclusion of the leptoquarkinos spoils the clear end point structure due to the presence of (at least) one lepton from the opposite ("wrong") side. A special remark is made here: if kinematical end points as the ones discussed above are distorted by a certain amount of events, which are positioned in a kinematically inaccessible region (i.e. beyond the edge), it may not necessarily mean that the combinatorics of a "typical" squark analysis went berserk, but rather indicate an underlying process, that has, as in our case, exotic fermions involved. A closer look onto a supplementary observable such as $m_{q l}^{*}$ from

\footnotetext{
${ }^{2}$ Mass scans are adjusted to take place from 600 to $1200 \mathrm{GeV}$.

${ }^{3}$ That is, they are to be applied on one side of the cascade only.
} 

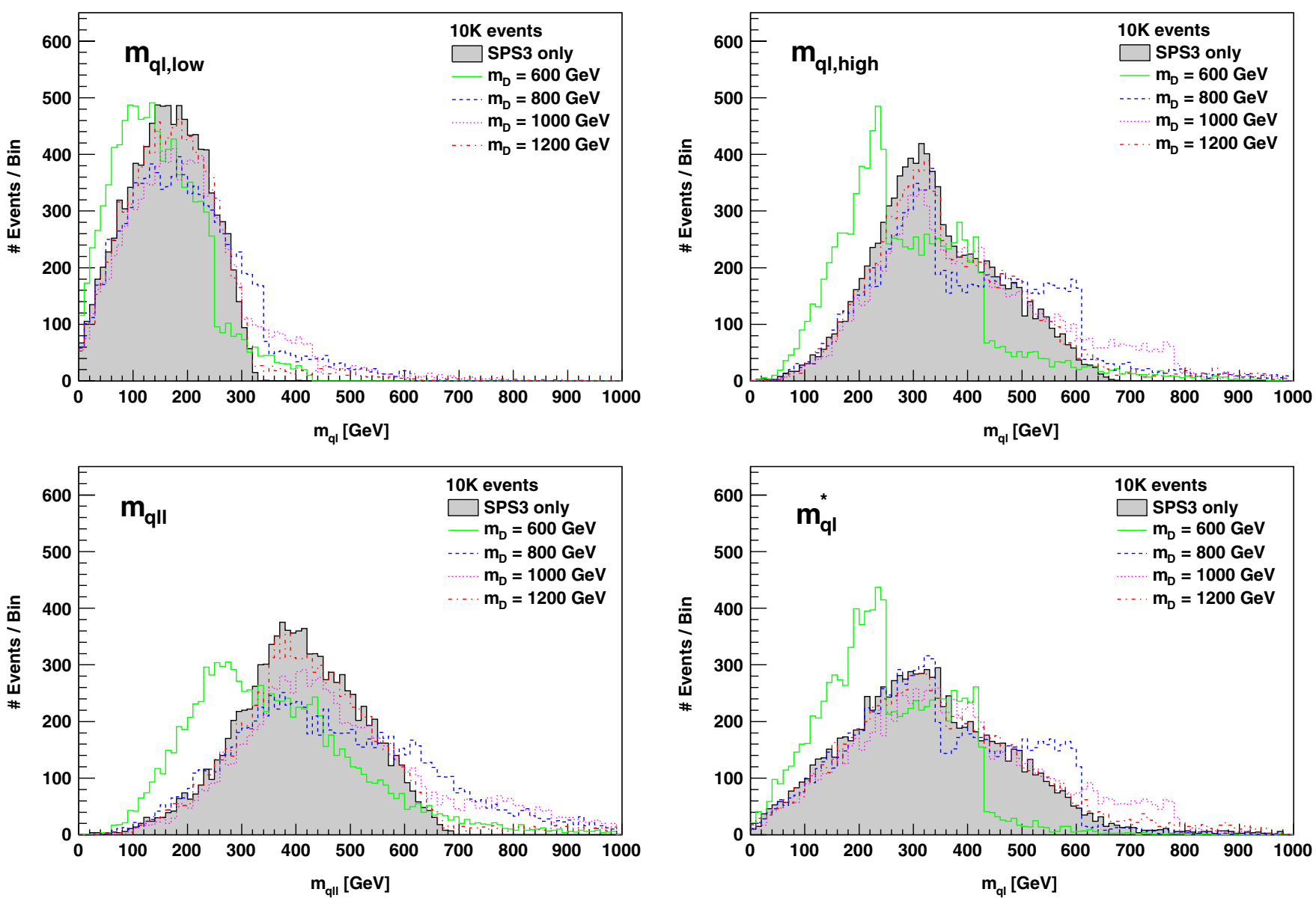

FIG. 7 (color online). Same as Fig. 6, but for SPS3.

Eq. (7), whose construction is free of combinatorical issues (which, however, could still suffer from an admixture from different decay sides), may then deliver additional insight into the specific process and thus the nature of the model in question.

Furthermore, considering one specific SPS scenario, namely, SPS1a, together with the extra exotic matter content, one is able to discriminate the squark and leptoquarkino signals by means of the two involved OSSF leptons in the following way: observation of the dileptonic invariant mass spectrum may indicate a kinematic end point for a vanilla MSSM signal, which would not be the case for a signature of leptoquarkinos. A rough significance estimate for $100 \mathrm{fb}^{-1}$ at $7 \mathrm{TeV}$ thus is possible by comparing the number of events for the sum of MSSM-type squark and leptoquarkino cascade to the ones who survive an analysis cut of $m_{l l}>100 \mathrm{GeV}$. The latter is based on the fact that the kinematical end point of the standard SUSY dilepton edge for underlying SPS1a is located below this fixed point in the invariant mass spectrum.

Significance estimates are given in Table I while Fig. 5 highlights the discrepancies between standard and nonstandard SUSY signals inherent in the dileptonic signal and the effect of the analysis cut on the variable $m_{l q}^{*}$ from Eq. (7) for a fixed exotic leptoquarkino mass of $m_{D}=600 \mathrm{GeV}$.

\section{CONCLUSIONS}

In this paper, we showed in the context of a quite general setup of GUT-inspired SUSY models containing nonstandard SUSY states how the physiognomy of standard kinematic variables for mass determination of cascade states can be altered. This happened as the lack of spin correlations does not distort the shape of those observables. However, the missing spin correlations do not come from a complete change of the underlying model paradigm (e.g. assuming UED) but from a slight variation or extension of the standard SUSY scenario. The potential for possible confusion in the model discrimination is specifically given in the case in which the corresponding scalar partners are too heavy to appear as resonances at the LHC. The significant issue of combinatorics may have a huge impact on the analysis, if interpreted in the wrong way. 

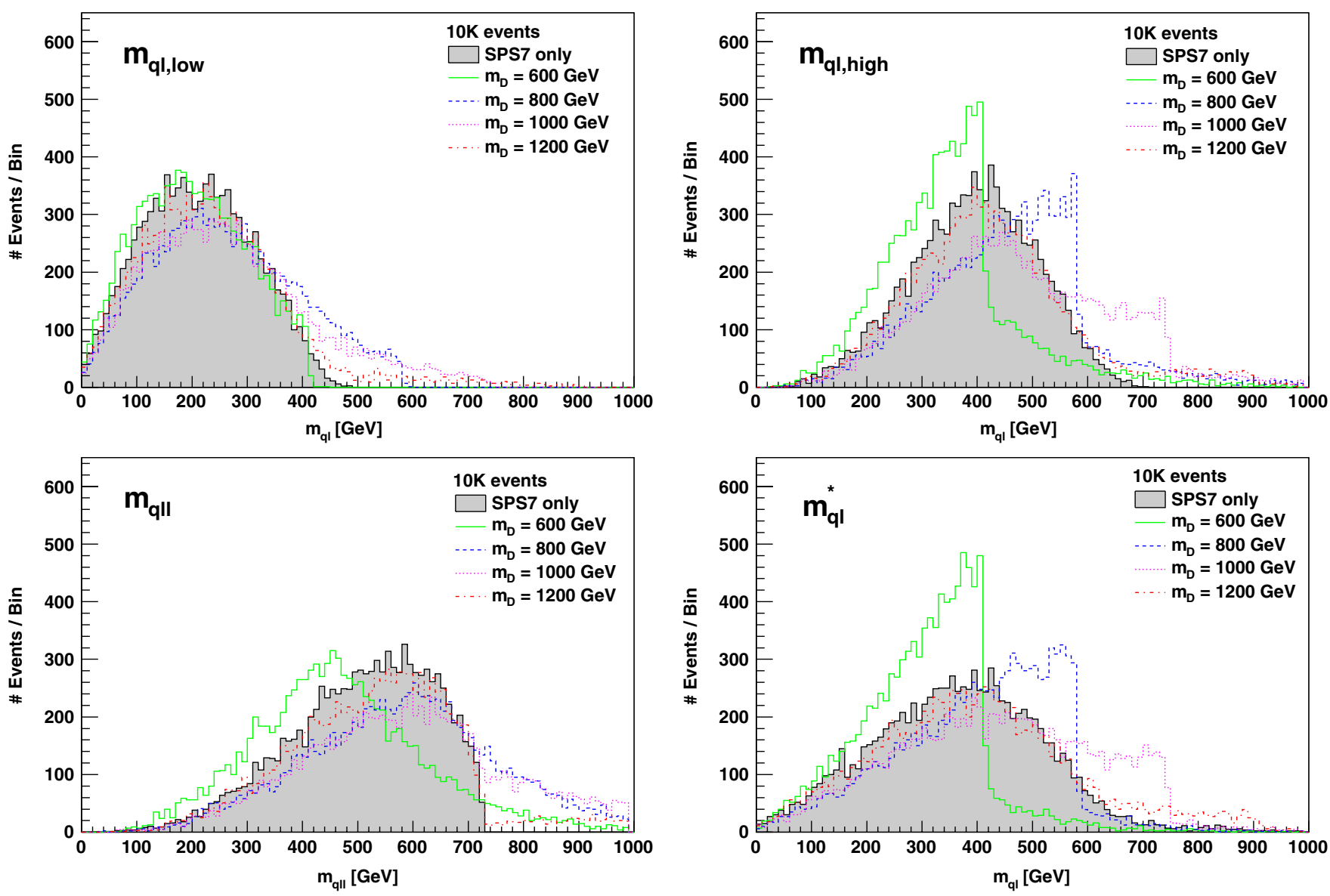

FIG. 8 (color online). Same as Figs. 6 and 7, but for SPS7.

Events in certain regions, which are kinematically forbidden in vanilla MSSM models, are prone to be dismissed. This comes about due to their false misidentification based on combinatorics and prevents a possible disclosure of a real signal from extended SUSY models or the like, in which the population of the aforementioned regions is inherent. Consequently this might serve as a prime example to show that model discrimination at the LHC is a crucial, but tedious task that needs to be carried out very carefully. The slightest variations of the underlying model assumptions may distort the shape and appearance of observables and likely misidentifications may furthermore even prevent the discovery of new, possibly exotic physics signals.

Leptoquarkinos in the context of SUSY models are a framework to consistently embed fermionic leptoquark states in a renormalizable model. These particles have, depending on their mass, rather high production rates at the LHC, and their smoking-gun signatures are very pronounced mass edges in several lepton-jet mass variables compared to standard squark/gluino mass edges. Other features such as mass pattern and flavor structure are more model dependent and not generic for these states. The discovery of leptoquarkinos could provide direct access to the GUT scale structure of supersymmetric field theories.

\section{ACKNOWLEDGMENTS}

We thank Alexander Knochel, Felix Jörder, and Christoph Horst for stimulating remarks, Felix Braam for providing us with his code, and Christian Speckner for help with the implementation. This work has been supported by the German Research Council (DFG) under Grant No. RE/2850/1-1 as well as by the Ministery for Research and Culture (MWK) of the German state BadenWürttemberg, and has also been partially supported by the DFG Graduiertenkolleg GRK 1102 "Physics at Hadron Colliders." D. W. acknowledges support from the Scottish Universities Physics Alliance (SUPA).

\section{APPENDIX: LEPTOQUARKINO EDGES FOR VARYING SUSY SPECTRA}

In this Appendix we collect the mass edge variables $m_{q l, \text { low }}, m_{q l \text {,high }}, m_{q l l}$, and $m_{q l}^{*}$ for varying SUSY spectra given by the Snowmass points SPS1b, SPS3, and SPS7, respectively, in Figs. 6-8. 
[1] J.L. Hewett and T. G. Rizzo, Phys. Rep. 183, 193 (1989).

[2] F. Braam, A. Knochel, and J. Reuter, J. High Energy Phys. 06 (2010) 013.

[3] F. Braam, C. Horst, A. Knochel, J. Reuter, and D. Wiesler (unpublished).

[4] C. G. Lester, Ph.D. thesis [Report No. CERN-THESIS2004-003, 2004].

[5] H. Bachacou, I. Hinchliffe, and F. E. Paige, Phys. Rev. D 62, 015009 (2000).

[6] C. Lester and A. Barr, J. High Energy Phys. 12 (2007) 102.

[7] G. Brooijmans et al., arXiv:1005.1229.

[8] A. J. Barr and C. G. Lester, J. Phys. G 37, 123001 (2010), and references therein.

[9] T. Plehn, D. Rainwater, and P.Z. Skands, Phys. Lett. B 645, 217 (2007).

[10] C. Athanasiou, C. G. Lester, J.M. Smillie, and B. R. Webber, J. High Energy Phys. 08 (2006) 055.

[11] B. C. Allanach et al., in Proceedings of the APS/DPF/DPB Summer Study on the Future of Particle Physics (Snowmass 2001), edited by N. Graf [Eur. Phys. J. C 25,
113 (2002)]; J. A. Aguilar-Saavedra et al., Eur. Phys. J. C 46, 43 (2006).

[12] W. Kilian, T. Ohl, and J. Reuter, arXiv:0708.4233; M. Moretti, T. Ohl, and J. Reuter, arXiv:hep-ph/0102195.

[13] A. Alboteanu, W. Kilian, and J. Reuter, J. High Energy Phys. 11 (2008) 010; J. Kalinowski, W. Kilian, J. Reuter, T. Robens, and K. Rolbiecki, J. High Energy Phys. 10 (2008) 090; T. Robens, J. Kalinowski, W. Kilian, J. Reuter, and K. Rolbiecki, Acta Phys. Pol. B 39, 1705 (2008) [http://th-www.if.uj.edu.pl/acta/vol39/abs/v39p1705.htm]; W. Kilian, J. Reuter, and T. Robens, Eur. Phys. J. C 48, 389 (2006); K. Hagiwara et al., Phys. Rev. D 73, 055005 (2006); M. Beyer et al., Eur. Phys. J. C 48, 353 (2006); W. Kilian, D. Rainwater, and J. Reuter, Phys. Rev. D 71, 015008 (2005); 74, 095003 (2006); T. Ohl and J. Reuter, Phys. Rev. D 70, 076007 (2004); Eur. Phys. J. C 30, 525 (2003).

[14] N. D. Christensen and C. Duhr, Comput. Phys. Commun. 180, 1614 (2009); N. D. Christensen, C. Duhr, B. Fuks, J. Reuter, and C. Speckner, arXiv:1010.3251.

[15] J. Kang, P. Langacker, and B. D. Nelson, Phys. Rev. D 77, 035003 (2008). 